\title{
BMJ Open Trauma airway management in emergency departments: a multicentre, prospective, observational study in Japan
}

\author{
Shunichiro Nakao, ${ }^{1}$ Akio Kimura, ${ }^{1}$ Yusuke Hagiwara, ${ }^{2}$ Kohei Hasegawa, ${ }^{3}$
} on behalf of the Japanese Emergency Medicine Network Investigators

To cite: Nakao S, Kimura $A$, Hagiwara $\mathrm{Y}$, et al. Trauma airway management in emergency departments: a multicentre, prospective, observational study in Japan. BMJ Open 2015;5:e006623. doi:10.1136/bmjopen-2014006623

- Prepublication history for this paper is available online. To view these files please visit the journal online (http://dx.doi.org/10.1136/ bmjopen-2014-006623).

Received 18 September 2014 Revised 19 December 2014 Accepted 22 December 2014

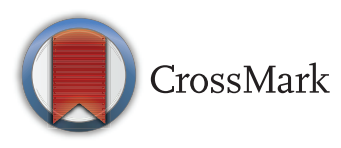

${ }^{1}$ Department of Emergency Medicine and Critical Care, National Center for Global Health and Medicine, Shinjuku, Tokyo, Japan ${ }^{2}$ Department of Emergency Medicine, Tokyo Metropolitan Children's Medical Center, Fuchu, Tokyo, Japan ${ }^{3}$ Department of Emergency Medicine, Massachusetts General Hospital, Harvard Medical School, Boston, Massachusetts, USA

Correspondence to Dr Shunichiro Nakao; shunichiro-nakao@umin.ac.jp

\section{ABSTRACT}

Objectives: Although successful airway management is essential for emergency trauma care, comprehensive studies are limited. We sought to characterise current trauma care practice of airway management in the emergency departments (EDs) in Japan.

Design: Analysis of data from a prospective, observational, multicentre registry-the Japanese Emergency Airway Network (JEAN) registry.

Setting: 13 academic and community EDs from different geographic regions across Japan.

Participants: 723 trauma patients who underwent emergency intubation from March 2010 through August 2012.

Outcome measures: ED characteristics, patient and operator demographics, methods of airway management, intubation success or failure at each attempt and adverse events.

Results: A total of 723 trauma patients who underwent emergency intubation were eligible for the analysis. Traumatic cardiac arrest comprised $32.6 \%$ $(95 \% \mathrm{Cl} 29.3 \%$ to $36.1 \%)$ of patients. Rapid sequence intubation (RSI) was the initial method chosen in $23.9 \%(95 \% \mathrm{Cl} 21.0 \%$ to $27.2 \%$ ) of all trauma patients and in $35.5 \%$ (95\% Cl $31.4 \%$ to $39.9 \%)$ of patients without cardiac arrest. Overall, intubation was successful in $\leq 3$ attempts in $96 \%$ of patients $(95 \% \mathrm{Cl}$ $94.3 \%$ to $97.2 \%$ ). There was a wide variation in the initial methods of intubation; RSI as the initial method was performed in $0-50.9 \%$ of all trauma patients among 12 EDs. Similarly, there was a wide variation in success rates and adverse event rates across the EDs. Success rates varied between $35.5 \%$ and $90.5 \%$ at the first attempt, and $85.1 \%$ and $100 \%$ within three attempts across the 12 EDs.

Conclusions: In this multicentre prospective study in Japan, we observed a high overall success rate in airway management during trauma care. However, the methods of intubation and success rates were highly variable among hospitals.

\section{INTRODUCTION}

Successful airway management is a cornerstone in the modern practice of emergency

\section{Strengths and limitations of this study}

- This is the first study to report disparities in trauma airway management based on multicentre, prospective data.

- Passive surveillance of data is subject to selfreporting bias, leading to a possible underestimation of failed intubations and adverse events.

and trauma care. Failure of emergency airway management is often associated with morbidity and mortality in trauma patients. ${ }^{1-3}$ Consequently, training in and understanding of airway management is a distinct discipline that is essential for successful trauma resuscitation.

Evidence-based recommendations for airway management during trauma care exist within international and national guidelines in the USA. These guidelines indicate rapid sequence intubation (RSI) as the initial method of emergency airway management in most trauma patients. ${ }^{1}{ }^{4} 5$ Recent studies reported that RSI is the most common airway management method in emergency departments (EDs) in North America and Europe. ${ }^{6-9}$ Despite the ubiquitous practice of emergency airway management in trauma patients, little is known about its current practice and performance in other industrialised nations. Therefore, we sought to describe the current practice of airway management for trauma patients in EDs in Japan.

\section{METHODS}

Study design and setting

We analysed the data of a prospective, observational, multicentre registry, the Japanese Emergency Airway Network registry. The study design, setting, methods of measurement and measured variables have been reported 
elsewhere. ${ }^{10-13}$ Briefly, the registry is a consortium of 13 academic and community EDs from different geographic regions across Japan. These EDs consist of 10 tertiary medical centres that have the capability of managing the most severe trauma patients and three secondary medical centres that are designated to treat moderately severe trauma patients. The participating EDs had a median ED census of 30000 patient visits per year (range 900067000 ). The participating hospitals had a median trauma admission of 1000 per year (range 300-1500). ${ }^{14}$ All 13 EDs were staffed by attending emergency physicians, and 12 had affiliations with emergency medicine residency training programmes. Each hospital maintained individual protocols, policies and procedures for emergency airway management. Intubations were performed by attending physicians or by resident physicians at the discretion of the ED's attending physician. The ethics committee of each participating centre approved the protocol, with waiver of informed consent before data collection.

\section{Patients}

The registry prospectively collected information on consecutive patients who underwent airway management in the participating EDs during a 30-month period, from March 2010 to August 2012. All adult and paediatric trauma patients who underwent intubation were eligible for this analysis. We excluded an ED in which the number of trauma intubations was less than 10 from the current analysis.

\section{Data collection}

Data collection was passive, relying on self-reports by the intubators on duty. After each intubation, the intubators completed a standardised data sheet, including the patient's age, sex, estimated weight, primary indication for intubation, methods used to facilitate intubation, intubator's level of training (emergency physicians, resident physicians and transitional-year residents) and specialty (emergency physician or not), number of attempts, success or failure, and intubation-related adverse events. Method was defined as the set of medications and devices used, such as RSI with a Macintosh laryngoscope. Transitional-year residents were postgraduate year 1-2 physicians who rotate through the EDs. An intubation attempt was defined as a single insertion of the laryngoscope (or other device) past the teeth. ${ }^{2}{ }^{10-13}$ For nasal intubations, an attempt was defined as a single insertion of a tracheal tube past the turbinates. An attempt was successful if it resulted in the tracheal tube being passed through the vocal cords. One or more methods could be used in each patient and each method could be attempted several times.

Adverse events were recorded using a prespecified list, with the option to include additional comments, if necessary. Adverse events were defined as cardiac arrest, hypotension, hypoxaemia, dysrhythmia, vomiting, oesophageal intubation, mainstem bronchial intubation and airway traumas that are considered to be intubation related. ${ }^{11}$ Cardiac arrest included asystole, bradycardia or dysrhythmia with non-measurable blood pressure and cardiopulmonary resuscitation during or after intubation. Hypotension was defined as systolic blood pressure less than $90 \mathrm{~mm} \mathrm{Hg}$. Hypoxaemia was defined as pulse oximetric saturation less than $90 \%$ during an intubation attempt, not a result of oesophageal intubation. Pre-existing hypotension or hypoxaemia before an intubation attempt was not counted as an adverse event. Oesophageal intubation was defined as misplacement of the endotracheal tube in the upper oesophagus or hypopharynx, with a lapse of time and desaturation (pulse oximetric saturation $<90 \%$ ) before the removal of the misplaced tube. We monitored compliance with data form completion by reviewing professional billing records. Where the data collection form was missing, the intubator was interviewed by one of the investigators within 2 weeks of the patient encounter, to fill out the data form.

The outcomes of interest were the primary indication for intubation, initial method used for intubation, intubation success rates (on the first attempt and within three attempts) and intubation-related adverse event rates.

\section{Statistical analysis}

We performed all analyses with JMP V.10 software (SAS Institute Inc, Cary, North Carolina, USA). At the patient level, we described patient demographics, the primary indication for intubation, initial method of intubation, success rates and adverse event rates as proportions with 95\% CIs and medians with IQRs. Then, at the ED level, we described medians, IQR and ranges for each outcome for all trauma patients. We also repeated the analysis after stratifying by indication (non-cardiac arrest vs cardiac arrest) and specialty (emergency physicians vs non-emergency physicians). All $\mathrm{p}$ values were two-tailed, with $\mathrm{p}<0.05$ considered statistically significant.

\section{RESULTS}

During the 30-month study period, 4268 patients required emergency airway management. Of these, the registry recorded 4094 intubations (capture rate, 95.9\%; figure 1), of which 3370 patients who underwent airway management for medical reasons were excluded from the analysis. One of the 13 hospitals, in which only one trauma patient required airway intervention during the study period, was excluded because the number of intubations for trauma care was less than 10 during the study period. Hence, 723 patients with trauma were eligible for analysis. Emergency physicians, including emergency medicine residents, performed the first intubation attempts in $60 \%$ (95\% CI $56.4 \%$ to $63.5 \%$ ) of all trauma patients and $66.7 \%$ (95\% CI $62.4 \%$ to $70.8 \%$ ) of patients without cardiac arrest. Transitional-year residents (postgraduate years 1 and 2) performed the first intubation attempts in $31.4 \%$ (95\% CI $28.1 \%$ to $34.9 \%)$ of all 


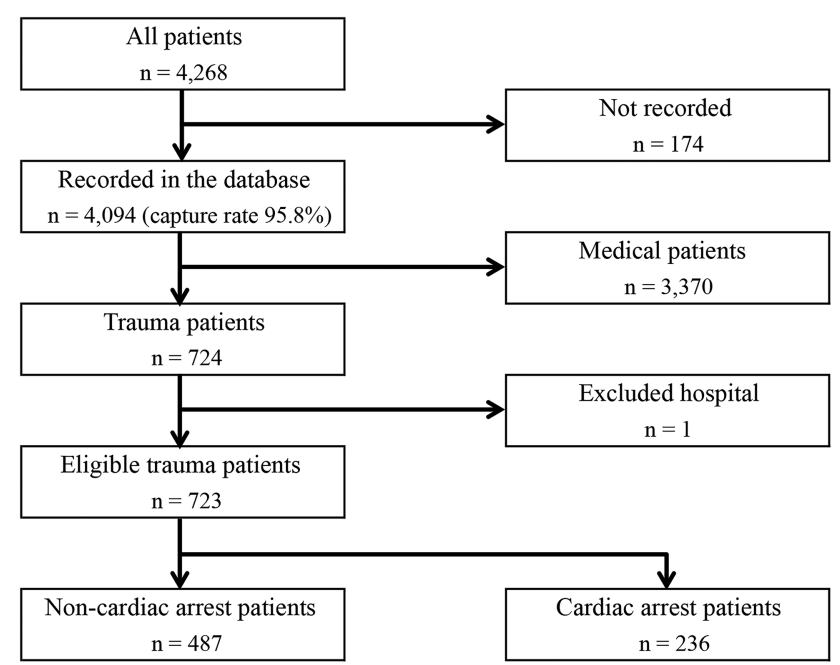

Figure 1 Flow chart showing inclusion of patients in this study.

trauma patients and $25.7 \%$ (95\% CI $22.0 \%$ to $29.7 \%$ ) of patients without cardiac arrest.

Table 1 shows the baseline characteristics and primary indications in patients who required trauma airway management. Median age was 56 years; two-thirds of the patients were male. Traumatic cardiac arrest was the reason for intubation in $32.6 \%$ (95\% CI $29.3 \%$ to $36.1 \%$ ) of all trauma patients, while head trauma accounted for $30.4 \%$ (95\% CI $27.2 \%$ to $33.9 \%$ ). Table 2 shows the initial method of airway management in the trauma patients. RSI was the initial method chosen in $23.9 \%(95 \%$ CI $21.0 \%$ to $27.2 \%)$ of all trauma patients and $35.5 \%$ (95\% CI $31.4 \%$ to $39.9 \%$ ) of patients without cardiac arrest. Cricothyrotomy was performed as the initial airway management strategy in $2.2 \%(95 \%$ CI $1.4 \%$ to $3.6 \%)$ of all trauma patients and $0.4 \%(95 \%$ CI $0.1 \%$ to $1.5 \%$ ) of patients without cardiac arrest. The direct laryngoscope was used in most intubations $(\mathrm{n}=654,90.5 \%)$ and the remaining were intubated using a video laryngoscope $(\mathrm{n}=30,4.1 \%)$, a bronchoscope $(\mathrm{n}=17,2.4 \%)$ or a lighted stylet $(\mathrm{n}=1,0.1 \%)$, on the first attempt.

\begin{tabular}{lll}
$\begin{array}{l}\text { Table } 1 \text { Characteristics of the } 723 \text { trauma patients who } \\
\text { required intubation }\end{array}$ & & \\
\hline Patient characteristics & & \\
Age (year), median (IQR) & 56 & $(34$ to 73$)$ \\
Male, \% (95\% Cl) & 66.9 & $(63.4$ to 70.3$)$ \\
Estimated weight $(\mathrm{kg})$, median (IQR) & 60 & $(50$ to 70$)$ \\
Indication for intubation, \% (95\% Cl) & & \\
Cardiac arrest & 32.6 & $(29.3$ to 36.1$)$ \\
Head trauma & 30.4 & $(27.2$ to 33.9$)$ \\
Shock & 16.6 & $(14.1$ to 19.5$)$ \\
Facial/neck trauma & 8.4 & $(6.6$ to 10.7$)$ \\
Airway burn & 6.8 & $(5.2$ to 8.8$)$ \\
Others & 5.1 & $(3.3$ to 8.0$)$ \\
\hline Cl, confidence interval; IQR, interquartile range. &
\end{tabular}

Table 3 summarises the intubation success rates and adverse event rates. Overall, intubation was successful in the first attempt in $63.8 \%$ (95\% CI $60.2 \%$ to $67.2 \%$ ) and within three attempts in $96 \%$ (95\% CI $94.3 \%$ to $97.2 \%$ ) of all trauma patients. In patients without cardiac arrest, intubation was successful in the first attempt in $60.2 \%$ (95\% CI $55.8 \%$ to $64.4 \%)$ and within three attempts in $94.5 \%$ (95\% CI $92.1 \%$ to $96.2 \%$ ) of patients. In patients with cardiac arrest, intubation was successful in the first attempt in $71.2 \%$ (95\% CI $65.1 \%$ to $76.6 \%$ ) and within three attempts in $99.2 \%$ (95\% CI $97.0 \%$ to $99.8 \%$ ) of the patients. In the stratified analysis by the specialty (ie, emergency physicians $(n=434)$ vs non-emergency physicians $(n=289))$, emergency physicians had a higher success at the first attempt $(72.8 \%$ vs $50.2 \%$, p $<0.001)$ compared to non-emergency physicians (including all transitional-year residents $(\mathrm{n}=237))$. Intubation-associated adverse event rates were $10.8 \%(95 \%$ CI $8.7 \%$ to $13.3 \%)$ in overall trauma patients, $11.5 \%(95 \%$ CI $9.0 \%$ to $14.6 \%)$ in patients without cardiac arrest and $9.3 \%$ (95\% CI $6.2 \%$ to $13.7 \%$ ) in patients with cardiac arrest.

At the ED level, there was a wide variation in the methods of intubation across the 12 EDs (figure 2). For example, RSI as the initial intubation method was performed in $0-50.9 \%$ of all trauma patients and in $0-87.5 \%$ of patients without cardiac arrest. Similarly, there was a wide variation in the success rates and adverse event rates across the EDs (figure 3). The range of overall success rates for intubation in the first attempt ranged from $35.5 \%$ to $90.5 \%$ and from $85.1 \%$ to $100 \%$ within three attempts. Likewise, overall adverse event rates varied widely (range $0-16.7 \%$ ) across the EDs. These wide variations in intubation success rates and adverse event rates persisted across the non-cardiac arrest and cardiac arrest strata.

\section{DISCUSSION}

In this prospective, multicentre, observational study in Japan, we observed an acceptable success rate of airway management in trauma patients in EDs. However, we also found a wide range of variation in the initial method of intubation, success rates and adverse event rates during trauma airway management across the EDs. Indeed, the overall success rates in the first intubation attempt ranged from $35.5 \%$ to $90.5 \%$.

We were struck by the high degree of variation in the methods of airway management in trauma cases across the 12 EDs. The reasons for the observed practice variations are unclear and are likely multifactorial. It is possible that non-RSI methods were attempted in patients who were predicted to have a difficult intubation. However, the difference in the patient population across the EDs cannot fully explain the observed threefold difference in the use of RSI. Therefore, some of the patients might have been inappropriately considered as 'difficult intubation' and intubated with non-RSI methods. Alternatively, it is also plausible that non-RSI 
Table 2 Initial method of intubation

\begin{tabular}{|c|c|c|c|c|c|c|}
\hline & \multicolumn{3}{|c|}{ All trauma patients } & \multicolumn{3}{|c|}{ Patients without cardiac arrest } \\
\hline & $\overline{\mathbf{n}}$ & Per cent & $95 \% \mathrm{Cl}$ & $\overline{\mathbf{n}}$ & Per cent & $95 \% \mathrm{Cl}$ \\
\hline Rapid sequence intubation & 173 & 23.9 & (21.0 to 27.2 ) & 173 & 35.5 & (31.4 to 39.9$)$ \\
\hline Sedation without paralysis & 153 & 21.2 & (18.3 to 24.3 ) & 153 & 31.4 & (27.5 to 35.7$)$ \\
\hline Paralytics without sedation & 19 & 2.6 & (1.7 to 4.1$)$ & 19 & 3.9 & (2.5 to 6.0$)$ \\
\hline Oral without sedation & 349 & 48.3 & (44.6 to 52.0$)$ & 127 & 26.1 & (22.4 to 30.2$)$ \\
\hline Surgical & 16 & 2.2 & (1.4 to 3.6$)$ & 2 & 0.4 & (0.1 to 1.5$)$ \\
\hline Nasal intubation & 13 & 1.8 & (1.1 to 3.1$)$ & 13 & 2.7 & (1.6 to 4.5$)$ \\
\hline Total & 723 & 100 & & 487 & 100 & \\
\hline
\end{tabular}

methods were more frequently used in certain EDs because of the physician's preference, procedural experiences, training background or differences in ED staffing and institutional policies.

Our study also demonstrated a high degree of variations in success and adverse event rates among the EDs. Particularly, the success rate at first attempt was highly variable. To the best of our knowledge, this is the first study to demonstrate such interhospital variations in success and adverse event rates in trauma airway management in different EDs. The reasons for the variations among the EDs are likely multifactorial; the potential explanations include interhospital differences in patient population, skills or education backgrounds of intubators, ${ }^{15}$ drug and device availability in the ED, or any combination of these factors. Alternatively, the observed wide variation in the intubation method may have led to these variations in success and adverse event rates. Furthermore, there are no requirements for procedural credentials to perform ED intubations in individuals as well as in institutions in Japan. ${ }^{10}$ This lack of procedural requirements would have contributed, at least partially, to the observed interhospital variations in the success rates. Indeed, we observed that intubation success rates performed by non-emergency physicians were significantly lower; this was, at least in part, explained by the intubation attempts by transitional-year residents. However, it is well documented in the literature that first-pass success is important in critically ill patients; ${ }^{11}$ therefore, the observed lower success rate by these nonskilled physicians cannot be justified. Our data underscore the need for reinforcement of the Japanese methodology of training for non-skilled physicians (eg, the use of simulators and training in a more controlled setting ${ }^{16-18}$ ) to improve their intubation skills, which will, in turn, improve patient outcomes.

Although international and Japanese trauma care guidelines recommend the use of RSI as the initial method of emergency airway management in most trauma patients, ${ }^{145}$ the evidence for accurately predicting patients in whom RSI should be avoided remains limited. ${ }^{19}{ }^{20}$ It is, therefore, plausible that the scarcity of evidence may have contributed to the practice variations across the EDs. Our observations should facilitate further investigation of any barriers to the delivery of safer trauma care nationally. Additionally, building more

Table 3 Success rates and intubation-associated adverse events

\begin{tabular}{|c|c|c|c|c|c|c|c|c|c|}
\hline & \multicolumn{3}{|c|}{ All trauma patients } & \multicolumn{3}{|c|}{$\begin{array}{l}\text { Patients without cardiac } \\
\text { arrest }\end{array}$} & \multicolumn{3}{|c|}{ Patients with cardiac arrest } \\
\hline & $\overline{\mathbf{n}}$ & Per cent & $95 \% \mathrm{Cl}$ & $\overline{\mathbf{n}}$ & Per cent & $95 \% \mathrm{Cl}$ & $\overline{\mathbf{n}}$ & Per cent & $95 \% \mathrm{Cl}$ \\
\hline Successful in 1st attempt & 461 & 63.8 & (60.2 to 67.2$)$ & 293 & 60.2 & (55.8 to 64.4$)$ & 168 & 71.2 & (65.1 to 76.6$)$ \\
\hline Successful in $\leq 3 r d$ attempts & 694 & 96.0 & (94.3 to 97.2$)$ & 460 & 94.5 & (92.1 to 96.2$)$ & 234 & 99.2 & (97.0 to 99.8$)$ \\
\hline Adverse events & 78 & 10.8 & (8.7 to 13.3$)$ & 56 & 11.5 & (9.0 to 14.6$)$ & 22 & 9.3 & (6.2 to 13.7$)$ \\
\hline \multicolumn{10}{|l|}{ Details of adverse events* } \\
\hline Oesophageal intubation & 25 & 3.5 & (2.2 to 5.1$)$ & 15 & 3.1 & (1.7 to 5.0$)$ & 10 & 4.2 & (2.0 to 7.7$)$ \\
\hline Mainstem bronchus intubation & 18 & 2.5 & (1.5 to 3.9$)$ & 9 & 1.8 & (0.8 to 3.5$)$ & 9 & 3.8 & (1.7 to 7.1$)$ \\
\hline Airway trauma & 17 & 2.4 & (1.4 to 3.7$)$ & 14 & 2.9 & (1.6 to 4.8$)$ & 3 & 1.3 & (0.3 to 3.7$)$ \\
\hline Hypotension† & 8 & 1.1 & (0.5 to 2.2 ) & 8 & 1.6 & (0.7 to 3.2$)$ & & & \\
\hline Vomiting & 6 & 0.8 & (0.3 to 1.8$)$ & 6 & 1.2 & (0.5 to 2.7 ) & & & \\
\hline Hypoxiał & 3 & 0.4 & (0.1 to 1.2$)$ & 3 & 0.6 & (0.1 to 1.8$)$ & & & \\
\hline Cardiac arrest & 1 & 0.1 & (0.0 to 0.8$)$ & 1 & 0.2 & (0.0 to 1.1$)$ & & & \\
\hline
\end{tabular}




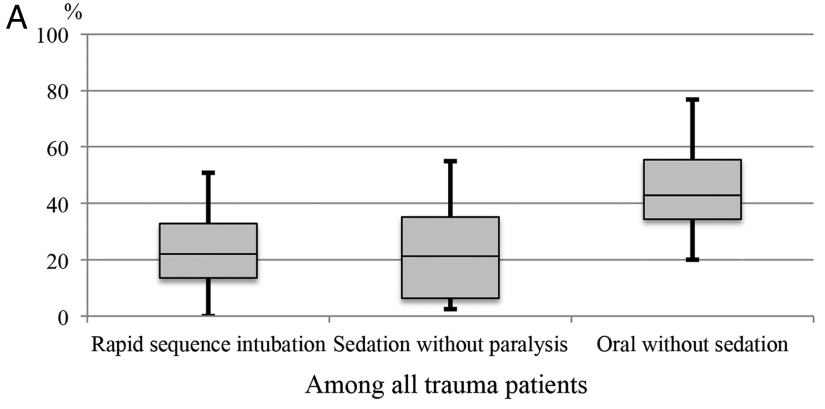

B

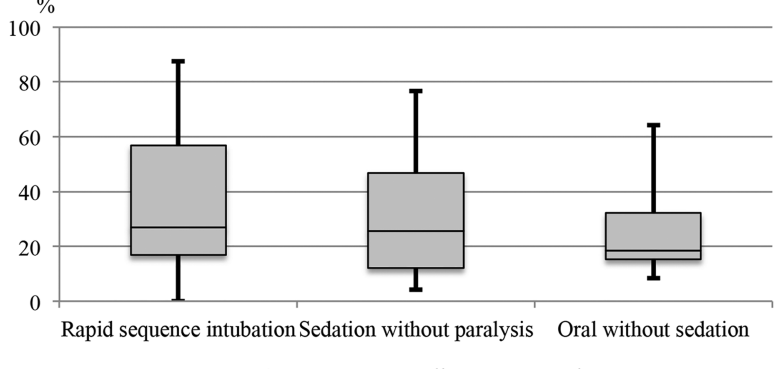

Among non-cardiac arrest patients

Figure 2 Interhospital variations in initial methods of intubation. Box plots of interhospital variations in the initial methods of intubation. The line in the middle of the box represents the median, with the lower and upper limits of the box representing the 25 th and 75 th centiles, respectively. The whiskers from the box extend to the minimum and maximum values.

robust evidence on trauma airway management, coupled with improved dissemination of these findings, could decrease the variations in trauma care across the EDs in Japan.

Our study has several potential limitations. First, this passive surveillance of the study data is subject to selfreporting bias, leading to a possible underestimation of failed intubations and adverse events. However, active independent monitoring of ED intubations is difficult to accomplish. We did, however, use a self-reporting system with structured data forms, uniform definitions and a high capture rate. Second, we did not design this study to measure patient outcomes, such as long-term mortality or morbidity. A more detailed analysis of adverse events and outcomes requires following the patients for a longer period. Third, we did not account for several potential confounders, such as severities (the Injury Severity Score, the Revised Trauma Score, etc) of cases and training levels of physicians. However, these prospective multicentre data reflect the current airway management in the natural setting of a 'real' population and current clinical practice, thereby enhancing the potential generalisability of the findings. Finally, all EDs in this study were designated as tertiary or academic general hospitals, and all but one of the EDs were affiliated with an emergency medicine residency programme in Japan. Therefore, our inferences may not be generalisable to trauma airway management in non-academic EDs or in other developed nations. These observations, however,

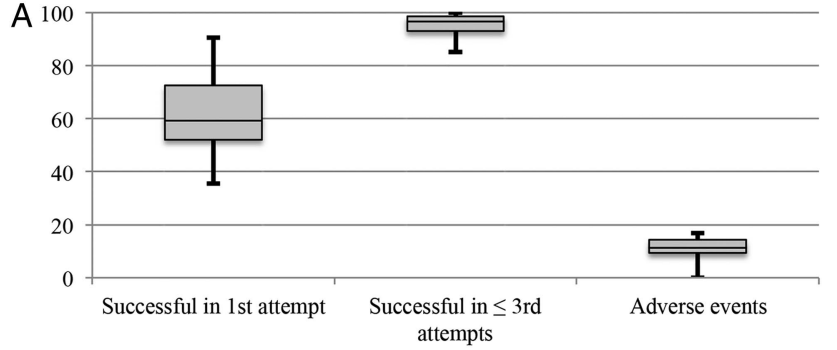

Among all trauma patients

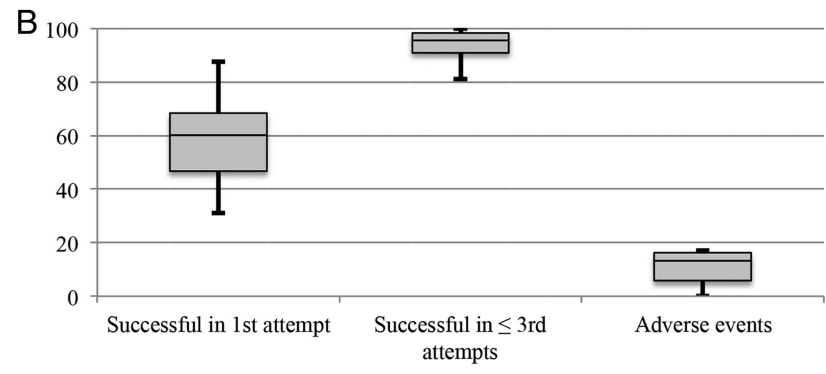

Among non-cardiac arrest patients

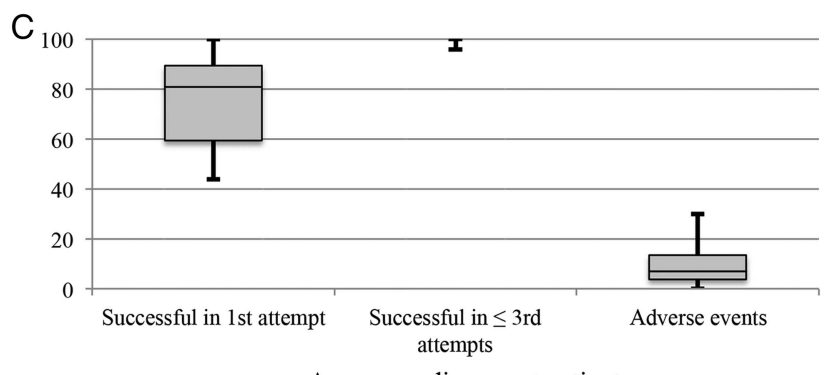

Among cardiac arrest patients

Figure 3 Interhospital variations in success rates and adverse event rates. Box plots of interhospital variations in success rates and adverse event rates. The line in the middle of the box represents the median, with the lower and upper limits of the box representing the 25th and 75th centiles. The whiskers from the box extend to the minimum and maximum values.

are highly relevant from a policy standpoint. As these EDs provide advanced care for trauma victims and train the majority of emergency physicians, they have a disproportionate impact on current and future trauma care in EDs in general.

\section{CONCLUSION}

In this multicentre prospective study of emergency airway management in Japan, we found an acceptable overall success rate in trauma airway management. However, we also found that the method of intubation, success rates and adverse event rates were highly variable among EDs. For researchers, our observations should facilitate further investigations to identify the reasons for the interhospital variations. Additionally, for policymakers and professional organisations, our findings suggest that development and dissemination of nationwide protocols are warranted to achieve safer airway management for trauma victims in Japan. 
Acknowledgements The authors acknowledge the following research personnel at the study hospitals for their assistance with this project: Fukui University Hospital (Hideya Nagai, MD, Hiroshi Morita, MD); Fukui Prefectural Hospital (Yukinori Kato, MD, Hidenori Higashi, MD); Japanese Red Cross Medical Center of Wakayama (Hiroshi Okamoto, MD); Kameda Medical Center (Kenzo Tanaka, MD); National Center for Global Health and Medicine (Taigo Sakamoto, MD, Shunichiro Nakao, MD); Nagoya Ekisaikai Hospital (Yukari Goto, MD); Niigata City General Hospital (Nobuhiro Sato, MD, MPH); Obama Municipal Hospital (Takuyo Chiba, MD); Okinawa Chubu Prefectural Hospital (Masashi Okubo, MD); Osaka Saiseikai Senri Hospital (Kazuaki Shigemitsu, MD); Shonan Kamakura General Hospital (Taichi Imamura, MD); Kurashiki Central Hospital (Hiroshi Okamoto, MD); and St. Marianna University School of Medicine Hospital (Yasuaki Koyama, MD). Finally, we are grateful to our many emergency physicians and residents for their perseverance in pursuing new knowledge about this vital resuscitative procedure.

Contributors $\mathrm{KH}$ designed the study, for which SN and YH contributed to the data collection and analysis. SN wrote the manuscript, which AK reviewed.

Funding This study was supported by a grant from St Luke's Life Science Institute and a grant from Massachusetts General Hospital and Brigham and Women's Hospital.

Competing interests None.

Ethics approval The ethics committee of each participating centre.

Provenance and peer review Not commissioned; externally peer reviewed.

Data sharing statement No additional data available.

Open Access This is an Open Access article distributed in accordance with the Creative Commons Attribution Non Commercial (CC BY-NC 4.0) license, which permits others to distribute, remix, adapt, build upon this work noncommercially, and license their derivative works on different terms, provided the original work is properly cited and the use is non-commercial. See: http:// creativecommons.org/licenses/by-nc/4.0/

\section{REFERENCES}

1. American College of Surgeons CoT. Advanced Trauma Life Support for Doctors, Student Course Manual. 8th ed. Chicago, IL, 2008.

2. Walls RM, Brown CA III, Bair AE, et al. Emergency airway management: a multi-center report of 8937 emergency department intubations. J Emerg Med 2011;41:347-54.

3. Hussain LM, Redmond AD. Are pre-hospital deaths from accidental injury preventable? BMJ 1994;308:1077-80.

4. Japanese Association for the Surgery of Trauma, Japanese Association of Acute Medicine. Japan Advanced Trauma Evaluation and Care (JATEC ${ }^{\mathrm{TM}}$ ) guideline. 3rd edn. Tokyo, Japan: Herusu Shuppan, 2008.

5. Dunham CM, Barraco RD, Clark DE, et al. Guidelines for emergency tracheal intubation immediately after traumatic injury. J Trauma 2003;55:162-79.

6. Graham CA. Emergency department airway management in the UK. $J$ S Soc Med 2005:98:107-10.

7. Sakles JC, Laurin EG, Rantapaa AA, et al. Airway management in the emergency department: a one-year study of 610 tracheal intubations. Ann Emerg Med 1998;31:325-32.

8. Sagarin MJ, Barton ED, Chng YM, et al. Airway management by US and Canadian emergency medicine residents: a multicenter analysis of more than 6,000 endotracheal intubation attempts. Ann Emerg Med 2005;46:328-36.

9. Graham CA, Beard D, Henry JM, et al. Rapid sequence intubation of trauma patients in Scotland. J Trauma 2004;56:1123-6.

10. Hasegawa K, Hagiwara Y, Chiba T, et al. Emergency airway management in Japan: interim analysis of a multi-center prospective observational study. Resuscitation 2012;83:428-33.

11. Hasegawa K, Shigemitsu K, Hagiwara $\mathrm{Y}$, et al. Association between repeated intubation attempts and adverse events in emergency departments: an analysis of a multicenter prospective observational study. Ann Emerg Med 2012;60:749-54 e2.

12. Imamura $\mathrm{T}$, Brown CA III, Ofuchi $\mathrm{H}$, et al. Emergency airway management in geriatric and younger patients: analysis of a multicenter prospective observational study. Am J Emerg Med 2013;31:190-6.

13. Hasegawa K, Hagiwara $Y$, Imamura $T$, et al. Increased incidence of hypotension in elderly patients who underwent emergency airway management: an analysis of a multi-centre prospective observational study. Int J Emerg Med 2013;6:12.

14. Hospital Intelligence Agency. Secondary Hospital Intelligence Agency. http://hospia.jp/

15. Graham CA, Beard D, Oglesby AJ, et al. Rapid sequence intubation in Scottish urban emergency departments. Emerg Med J 2003;20:3-5.

16. Mayo PH, Hackney JE, Mueck JT, et al. Achieving house staff competence in emergency airway management: results of a teaching program using a computerized patient simulator. Crit Care Med 2004;32:2422-7.

17. Cook DA, Hatala R, Brydges R, et al. Technology-enhanced simulation for health professions education: a systematic review and meta-analysis. JAMA 2011;306:978-88.

18. Bernhard M, Mohr S, Weigand MA, et al. Developing the skill of endotracheal intubation: implication for emergency medicine. Acta Anaesthesiol Scand 2012;56:164-71.

19. Reed MJ, Dunn MJ, McKeown DW. Can an airway assessment score predict difficulty at intubation in the emergency department? Emerg Med J 2005;22:99-102.

20. Soyuncu S, Eken C, Cete Y, et al. Determination of difficult intubation in the ED. Am J Emerg Med 2009;27:905-10. 